\title{
SPACES OF CONSTANCY OF CURVATURE OPERATORS ${ }^{1}$
}

\section{ALFRED GRAY}

1. Introduction. There are three kinds of Riemannian manifolds whose study is facilitated by the fact that their curvature operators have a particularly nice form; these are flat spaces, spaces of constant [curvature, and spaces of constant] holomorphic curvature. It is natural, therefore, on a general Riemannian manifold $M$ to study the distribution which assigns to each $m \in M$ the subspace of the tangent space $M_{m}$ to $M$ at $m$ on which the curvature operator behaves like one of the above types. For example, let $R(m)=$ $\left\{x \in M_{m} \mid R_{x y}=0\right.$ for all $\left.y \in M_{m}\right\}$, where $R_{x y}$ denotes the curvature operator. Chern and Kuiper [1] have proved that the distribution $m \rightarrow R(m)$ is integrable and its integral manifolds are flat. Maltz [4] has shown that the integral manifolds are totally geodesic and investigated their completeness properties.

The subspace $R(m)$ of $M_{m}$ is called the space of nullity of the curvature operator at $m$. Similarly we define the space of constancy $R_{K}(m)$ and the space of holomorphic constancy $\mathfrak{H}_{K}(m)$ of the curvature operator at $m$. Here $\mathcal{R}_{K}(m)$ is the subspace of $M_{m}$ on which the curvature operator has constant curvature $K$, and $\mathfrak{F}_{K}(m)$ is the subspace on which it has constant holomorphic curvature $4 K$. We prove that the distributions $m \rightarrow \mathfrak{R}_{K}(m)$ and $m \rightarrow \mathfrak{H C}_{K}(m)$ are integrable. The integral submanifolds are totally geodesic, have constant or constant holomorphic curvature and possess the same completeness properties as in the flat case. Ôtsuki [6] has also considered the spaces of constancy $\mathbb{R}_{K(m)}$.

2. Riemannian tensors. Let $M$ be a Riemannian manifold of class $C^{\infty}$; we denote by $\mathcal{F}(M)$ the ring of differentiable real-valued functions on $M$ and by $\mathfrak{X}(M)$ its derivation Lie algebra, which consists of the vector fields on $M$. The metric tensor field will be denoted by $\langle$,$\rangle , the Riemannian connection by \nabla_{X}(X \in \mathfrak{X}(M))$, and the curvature operator by $R_{X Y}(X, Y \in \mathfrak{X}(M))$. If $M$ is almost complex $J: \mathfrak{X}(M)$ $\rightarrow \mathfrak{X}(M)$ will denote the almost complex structure. Since $\langle\rangle,$,$R , and$ $J$ are tensor fields, they determine tensors on each tangent space, which we denote by the same symbols. In order to unify our proofs we consider a special class of $(1,3)$ tensor fields.

Received by the editors October 4, 1965.

1 This work was supported in part by the National Science Foundation under Grant GP-3990. 
(2.1) Definition. A Riemannian tensor field on $M$ is a tensor field $A$ of type $(1,3)$, which for $X, Y \in \mathfrak{X}(M)$ we regard as an $\mathcal{F}(M)$-linear map $A_{X Y}: \mathfrak{X}(M) \rightarrow \mathfrak{X}(M)$. It is required to have the following properties:

(2.1.1) $A_{X Y}=-A_{Y X}$

(2.1.2) $\left\langle A_{X Y}(Z), W\right\rangle=-\left\langle A_{X Y}(W), Z\right\rangle$,

(2.1.3) $\subseteq A_{X Y}(Z)=0$,

(2.1.4) $\subseteq \nabla_{X}(A)_{Y Z}=0$,

for $X, Y, Z, W \in \mathfrak{X}(M)$, where $\mathfrak{S}$ denotes the cyclic sum. If $\nabla_{X}(A)_{Y Z}$ $=0$ for all $X, Y, Z \in \mathfrak{X}(M)$ we say that $A$ is parallel, and if $\nabla_{X}(A)$ $=\alpha(X) A$ for some 1 -form $\alpha$ we say that $A$ is recurrent.

It is easy to see that (2.1.1)-(2.1.3) imply

(2.1.5) $\left\langle A_{X Y}(Z), W\right\rangle=\left\langle A_{Z W}(X), Y\right\rangle$ for $X, Y, Z, W \in \mathfrak{X}(M)$. If $\left\langle A_{X Y}(X), Y\right\rangle=0$ for all $X, Y \in \mathfrak{X}(M)$, then $A=0$ (see Helgason $[3$, p. 68]). Also, it is clear that the Riemannian tensor fields are closed under addition and multiplication by real numbers.

(2.2) Lemma. Let $A$ be a Riemannian tensor. Then for $X, Y, Z$ $\in \mathfrak{X}(M)$ we have

$$
\Im\left\{\left[\nabla_{X}, A_{Y Z}\right]-A_{[X, Y] Z}\right\}=0 .
$$

Proof. We have $\nabla_{X}(Y)-\nabla_{Y}(X)=[X, Y]$ for $X, Y \in \mathfrak{X}(M)$, and so

$$
\begin{aligned}
0 & =\Im \nabla_{X}(A)_{Y Z}=\mathfrak{S}\left\{\left[\nabla_{X}, A_{Y Z}\right]-A_{\nabla_{X}(Y) Z}-A_{Y_{\nabla_{Y}}(Z)}\right\} \\
& =\mathfrak{S}\left\{\left[\nabla_{X}, A_{Y Z}\right]-A_{[X, Y] Z}\right\} .
\end{aligned}
$$

(2.3) Definition. Let $m \in M$. We define

$$
a(m)=\left\{x \in M_{m} \mid A_{x y}=0 \text { for all } y \in M_{m}\right\},
$$

and we denote by $Q$ the distribution $m \rightarrow Q(m)$. (Here $A_{x y}$ is the operator on $M_{m}$ determined by $A$.) We call $Q(m)$ the space of nullity of $A$ at $m, a$ the field of nullity of $A$, and $\operatorname{dim} Q(m)$ the index of nullity of $A$ at $m$.

(2.4) Theorem. Let $U$ be an open subset of $M$ on which the index of nullity of $A$ is constant. Then the distribution $Q$ is integrable on $U$.

Proof. Let $X$ and $Y$ be a vector fields in $a$. From (2.2) it follows that $[X, Y]$ is in $Q$.

It is clear that the curvature operator is a Riemannian tensor field; however, there are two other Riemannian tensors that we shall be particularly interested in, namely $B$ and $D$, defined as follows:

$$
B_{X Y}(Z)=R_{X Y}(Z)-K(\langle X, Z\rangle Y-\langle Y, Z\rangle X) .
$$




$$
\begin{aligned}
D_{X Y}(Z)= & R_{X Y}(Z)-K(\langle X, Z\rangle Y-\langle Y, Z\rangle X \\
& +\langle J X, Z\rangle J Y-\langle J Y, Z\rangle J X+2\langle J X, Y\rangle J Z),
\end{aligned}
$$

for $X, Y, Z \in \mathfrak{X}(M)$, where $K$ is a constant. The latter tensor field is defined if $M$ is almost complex. It is not hard to verify that $B$ is Riemannian, and if $M$ is Kählerian, $D$ is Riemannian. It can be shown [3], [7] that $B=0$ if and only if $M$ has constant curvature $K$, and that $D=0$ if and only if $M$ has constant holomorphic curvature $4 K$.

We denote by $R_{K}$ and $\mathfrak{F}_{K}$ the fields of nullity of $B$ and $D$ respectively, and we call $\Re_{K}(m)$ and $\mathfrak{H}_{K}(m)$ the spaces of constancy and holomorphic constancy of the curvature operator at $m$. We shall be concerned only with the tensor fields $B$ and $D$; however, there are other interesting Riemannian tensor fields. For example, if $M$ is locally symmetric (i.e., the curvature operator is parallel), the Weyl conformal tensor field is a parallel Riemannian tensor field, and by (2.4) its field of nullity is integrable.

3. Local properties of the integral manifolds. Let $L$ be a Riemannian manifold isometrically imbedded in another Riemannian manifold $M$. Let $\overline{\mathfrak{X}}(L)=\{X|L| X \in \mathfrak{X}(M)\}$; then we write $\overline{\mathfrak{X}}(L)=\mathfrak{X}(L)$ $\oplus \mathfrak{X}(L) \perp$ where $\mathfrak{X}(L) \perp$ is the collection of vector fields normal to $L$. Let $P: \overline{\mathfrak{X}}(L) \rightarrow \mathfrak{X}(L)$ be the natural projection. For $X, Y \in \mathfrak{X}(L)$ we denote the Riemannian connection and curvature operator of $L$ by $\delta_{X}$ and $r_{X Y}$ respectively. The configuration tensor [2] of $L$ in $M$ is an $\mathcal{F}(M)$-linear map $t: \mathfrak{X}(L) \times \overline{\mathfrak{X}}(L) \rightarrow \overline{\mathfrak{X}}(L)$ defined by $t_{X}(Y)=\nabla_{X}(Y)$ $-\delta_{X}(Y)(X, Y \in \mathfrak{X}(L))$ and $t_{X}(Z)=P \nabla_{X}(Z)(X \in \mathfrak{X}(L), Z \in \mathfrak{X}(L) \perp)$. Then $t_{X}(\mathfrak{X}(L)) \subseteq \mathfrak{X}(L) \perp, t_{X}(\mathfrak{X}(L) \perp) \subseteq \mathfrak{X}(L)$ for $X \in \mathfrak{X}(L), t_{X}(Y)=t_{Y}(X)$ for $X, Y \in \mathfrak{X}(L)$ and $\left\langle t_{X}(Z), W\right\rangle=-\left\langle t_{X}(W), Z\right\rangle$ for $W, X \in \mathfrak{X}(L)$, $Z \in \mathfrak{X}(L) \perp$. The configuration tensor vanishes if it vanishes on either $\mathfrak{X}(L)$ or $\mathfrak{X}(L)^{\perp}$, and so it is equivalent to the second fundamental form, which in our terminology would be the map $X \rightarrow t_{X}(Z)$ for $X \in \mathfrak{X}(L), Z \in \mathfrak{X}(L) \perp$.

We now prove that the field of nullity of a Riemannian tensor is totally geodesic. First we state a lemma, the proof of which is obvious.

(3.1) Lemma. Let $L$ be an integral manifold of $a$. Then if $X, Y, Z$ $\in \mathfrak{X}(L)^{\perp}, A_{X Y}(Z) \in \mathfrak{X}(L)^{\perp}$.

(3.2) Theorem. Let $L$ be an integral manifold of $a$; then $L$ is totally geodesic.

Proof. Let $X \in \mathfrak{X}(L)$ and $Y, Z, U \in \mathfrak{X}(L) \perp$. We first show that $t_{X}\left(A_{Y Z}(U)\right)=0$. Since $A_{Y Z}(U) \in \mathfrak{X}(L) \perp$ we have 


$$
\begin{aligned}
P \Im_{X Y Z} \nabla_{X}\left(A_{Y Z}(U)\right) & =P\left\{\nabla_{X}\left(A_{Y Z}(U)\right)+\nabla_{Z}\left(A_{X Y}(U)\right)+\nabla_{Y}\left(A_{Z X}(U)\right)\right\} \\
& =t_{X}\left(A_{Y Z}(U)\right) .
\end{aligned}
$$

On the other hand by (2.2) and (3.1)

$$
\begin{aligned}
P \Im_{X Y Z} \nabla_{X}\left(A_{Y Z}(U)\right) & =P \Im\left\{A_{Y Z}\left(\nabla_{X}(U)\right)+A_{[X, Y] Z}(U)\right\} \\
& =A_{Y Z} P \nabla_{X}(U)+A_{P[X, Y] Z}(U)+A_{P[Z, X] Y}(U) \\
& =0 .
\end{aligned}
$$

Next we let $W \in \mathfrak{X}(L)$. Then $t_{X}(W) \in \mathfrak{X}(L) \perp$ and so by (3.1) $A_{Y Z}\left(t_{X}(W)\right) \in \mathfrak{X}(L) \perp^{\perp}$; however, $\left\langle A_{Y Z}\left(t_{X}(W)\right), U\right\rangle=\left\langle W, t_{X}\left(A_{Y Z}(U)\right)\right\rangle$ $=0$ and so $t_{X}(W) \in \mathfrak{X}(L)$. Hence $t_{X}(W)=0$; this proves that $L$ is totally geodesic.

(3.3) Corollary. Suppose $L$ is an integral manifold of $\mathfrak{R}_{K}$ or $\mathfrak{H}_{K}$. Then $L$ has constant curvature $K$ or constant holomorphic curvature $4 K$. If $M$ is a Kähler manifold and $M$ is an integral manifold of $\mathfrak{H}_{K}$, then $L$ is a Kähler submanifold of $M$.

Proof. The first statement follows from the Gauss equation (see [2]):

$$
P R_{X Y}=r_{X Y}-\left[t_{X}, t_{Y}\right] \text {. }
$$

For the last statement let $X \in \mathfrak{X}(L), Y \in \overline{\mathfrak{X}}(L)$. Then

$$
D_{J X Y}=J D_{X Y}=0,
$$

and so $J X \in \mathfrak{X}(L)$; hence $L$ is a Kähler submanifold of $M$.

4. The completeness of the integral manifolds. Let $G$ be the set on which the index of nullity $\mu$ assumes its minimum value $\lambda$.

(4.1) Proposition. The function $\mu$ is upper semicontinuous, and the set $G$ is open.

Proof. It suffices to prove that for any $m \in M$ there exists a neighborhood $U$ of $m$ such that $\mu(p) \leqq \mu(m)$ for $p \in U$, but this is obvious.

We shall need the following lemma. Let $n=\operatorname{dimension}$ of $M$.

(4.2) Lemma. Let $\gamma:[0, b) \rightarrow L$ be a unit speed geodesic in an integral manifold $L$ of $Q$ in $G$. Then there exists a frame field $\left\{e^{1}, \cdots, e^{n}\right\}$ on $\gamma$ such that:

(4.2.1) $e_{\gamma(t)}^{i} \in a(\gamma(t))(1 \leqq i \leqq \lambda, t \in[0, b))$.

(4.2.2) $\gamma^{\prime}(t)=e_{\gamma(t)}^{1}(t \in[0, b))$.

(4.2.3) The frame field is parallel on $\gamma$. 
Proof. We may assume (4.2.1)-(4.2.3) hold for $t=0$. The frame field is defined at an arbitrary $t \in[0, b)$ by parallel translation. It is obvious that (4.2.2) and (4.2.3) are satisfied, and (4.2.1) holds because the parallel translation takes place along the submanifold $L$.

(4.3) Theorem. Assume $M$ is complete and that $A$ is recurrent. Then each integral manifold $L$ of $Q$ in $G$ is complete.

Proof. If $\lambda=n$ the proof is trivial, so we assume $\lambda<n$. Let $\gamma:[0, b)$ $\rightarrow L$ be a unit speed geodesic. Since $M$ is complete we may extend $\gamma$ to a geodesic $\gamma:[0, \infty) \rightarrow M$. Let $\left\{e^{1}, \cdots, e^{n}\right\}$ be a frame field on $\gamma \mid[0, b)$ which satisfies (4.2.1)-(4.2.3). Then we may define $\left\{e_{1}, \cdots, e_{n}\right\}$ at $\gamma(b)$ by parallel translation; (4.2.1)-(4.2.3) now hold for $\gamma \mid[0, b]$. We extend each $e^{i}$ to a vector field $E^{i}$ defined on a neighborhood $N$ of $\gamma \mid[0, b]$. Also, let $X, Y$ be vector fields on $N$ such that $X_{\gamma(t)}, Y_{\gamma(t)} \in Q(\gamma(t)) \perp$ for $t \in[0, b)$ and $X, Y$ are parallel on $\gamma \mid[0, b]$.

Let $\lambda+1 \leqq p, q, r \leqq n$; we define $\Phi_{p q}:[0, b] \rightarrow R$ and $\Gamma_{p q}:[0, b] \rightarrow R$ by

$$
\Phi_{p q}=\left\langle A_{E^{p} E^{q}}(X), Y\right\rangle \circ \gamma
$$

By (2.1.4) and (4.2.1)-(4.2.3) it follows that

$$
\stackrel{\dot{\Phi_{p q}}}{\prime}=0
$$

Since the matrix $\left(\Phi_{p q}\right)$ is nonzero at 0 , it follows from the theory of ordinary differential equations that $\left(\Phi_{p q}\right)$ cannot vanish at $b$. The vector fields $X$ and $Y$ are arbitrary and so $\mu(\gamma(b))=\lambda$. Therefore $\gamma(b) \in G$ and so there exists $c>b$ such that $\gamma([0, c)) \subset G$. Hence every geodesic in $L$ is infinitely extendable (in $L$ ) and so $L$ is complete.

5. Some examples. (a) Consider the "dishpan surface," the graph of the function $f: R^{2} \rightarrow R$ defined by

$$
f(x, y)= \begin{cases}-\exp \left(\frac{+1}{x^{2}+y^{2}-r^{2}}\right), & x^{2}+y^{2}<r^{2}, \\ 0, & x^{2}+y^{2} \geqq r^{2},\end{cases}
$$

where $0 \leqq r<\infty$. The index of nullity $\mu$ of the curvature operator of this surface is 0 on $G=\left\{(x, y) \mid x^{2}+y^{2}<r^{2}\right\}$ except for one circle and 2 on the complement of $G$. By choosing the function $f$ differently the set $G$ can be made to assume a variety of shapes; for example, polygonal regions or the complement of a finite set. 
(b) The index of nullity of the Cartesian product of $n$ dishpan surfaces assumes the values $0,2, \cdots, 2 n$.

(c) Let $F$ be a flat manifold of dimension $k$ : for example, $R^{k}$ or a $k$-dimensional flat torus $T^{k}$. If the index of nullity of a manifold $M$ assumes its minimum value $\lambda$ on $G \subset M$, then the index of nullity of the curvature operator assumes its minimum value $\lambda+k$ on $G \times F$. If $\lambda=0$, the folliation of $G \times F$ consists of manifolds of the form $\{p\} \times F$ $(p \in M)$.

(d) Let $M$ be an $n$-dimensional manifold and let $Q$ be $R_{K}$ or $\mathfrak{H}_{K}$, where $K \neq 0$. The author conjectures that the minimum value of the index of nullity of $Q$ is either 0 or $n$.

In the preparation of this paper the author has found conversations with B. O'Neill and R. Maltz helpful.

\section{REFERENCES}

1. S. S. Chern and N. H. Kuiper, Some theorems on the isometric imbedding of compact Riemannian manifolds in Euclidean space, Ann. of Math. (2) 56 (1952), 422-430.

2. A. Gray, Minimal varieties and almost Hermitian submanifolds, Mich. Math. J. 12 (1965), 273-287.

3. S. Helgason, Differential geometry and symmetric spaces. Academic Press, New York, 1958.

4. R. Maltz, The nullity spaces of the curvature operator, Thesis, University of California, Los Angeles, 1965.

5. B. O'Neill and E. Stiel, Isometric immersions of constant curvature manifolds, Mich. Math. J. 10 (1963), 335-339.

6. T. Ôtsuki, Isometric imbedding of Riemann manifolds in a Riemann maniford, J. Math. Soc. Japan 6 (1954), 221-234.

7. K. Yano, The theory of Lie derivatives and its applications, North-Holland, Amsterdam, 1957.

University of California, Berkeley 